\title{
7. Ontologische Implikationen des Stringansatzes
}

Einer wissenschaftsphilosophischen Auseinandersetzung mit dem Stringansatz kommt, wie zuvor schon erörtert, ${ }^{365}$ notwendigerweise bis zu einem gewissen Grade der Status der Vorläufigkeit zu. Dieser wird jedoch angesichts der Bedeutung und der langanhaltenden, über diverse Metamorphosen hinweg fortschreitenden Entwicklungsgeschichte des Stringansatzes nicht dahingehend wirksam, eine solche wissenschaftsphilo-sophische Auseinandersetzung bis auf irgendeinen zukünftigen Tag des Hervortretens einer endgültigen, abgeschlossenen Theorie zu verschieben. Aber dieser Status der Vorläufigkeit trifft vermutlich keinen Bereich einer wissenschaftsphilosophischen Auseinandersetzung mit dem Stringansatz stärker, als die Frage nach seinen ontologischen Implikationen und die nach seiner Stellung zur Realismusproblematik.

"Ultimately, the biggest barrier to the construction of a theory of quantum gravity may not be the mathematics, but the interpretation of the mathematics. "(Matthews (1994) 32)

Auch wenn es angesichts der Tatsache, dass es eigentlich keine Theorie und keine fundamentalen Prinzipien gibt - und erst recht keine empirischen Belege -, sicherlich noch erheblich verfrüht sein dürfte, über die ontologischen Implikationen des Stringansatzes zu entscheiden, so ist es vielleicht dennoch nicht zu früh, zumindest einmal über die diesbezüglichen Möglichkeiten nachzudenken.

\section{Sind Strings fundamentale Entitäten?}

Hinsichtlich der Erörterung des ontischen Status bzw. der realistischen Deutbarkeit theoretisch postulierter Entitäten stellt sich erst einmal die

$365 \quad$ Siehe die Einleitung zu Kap. 4. 
Frage, welche fundamentalen Entitäten der Stringansatz überhaupt postuliert. Für die perturbativen Stringtheorien sind dies zwar recht unzweifelhaft die Strings selbst, die sich auf einer statischen zehndimensionalen Hintergrundraumzeit bewegen und schwingen. Aber beim Übergang vom perturbativen in den nicht-perturbativen Bereich der von den Stringtheorien beschriebenen Dynamik, für den es immerhin schon tentative Ansätze gibt, wird dieses Bild in erheblicher Weise in Frage gestellt. Es sind nicht zuletzt die Dualitäten zwischen den verschiedenen perturbativen Stringtheorien und die in diesen Dualitäten aufschimmernden Einsichten in den nicht-perturbativen Bereich, welche die Festlegung der fundamentalen Entitäten uneindeutig werden lassen.

"Often under the duality map, an elementary particle in one theory gets mapped to a composite particle in a dual theory and vice versa. Thus classification of particles into elementary and composite loses significance as it depends on which particular theory we use to describe the system." (Sen (1998) 2)

Theorien, deren fundamentale dynamische Entitäten, entsprechend dem perturbativen Ansatz, offensichtlich Strings sind, erweisen sich für bestimmte Parameterbereiche als dual, und damit als hinsichtlich ihrer phänomenologisch relevanten Ergebnisse äquivalent, zu Theorien, deren fundamentale Entitäten scheinbar Branen und weitere solitonische Zustände sind.

"In addition to the fundamental strings, various string theories have in their spectra one-dimensional objects which are either smooth solitons or D-branes. At weak coupling these are much heavier than the fundamental strings, but at strong coupling they are much lighter (again this guaranteed by the BPS formula). In this limit it is natural to reinterpret the theory with the soliton or D-brane being the fundamental string." (Polchinski (1996) 22)

In verschiedenen Parameterbereichen und unter verschiedenen Kompaktifizierungsbedingungen für die überzähligen Dimensionen können die unterschiedlichsten Objekte (bzw. mathematischen Konstrukte) die Rolle der fundamentalen dynamischen Entitäten spielen. ${ }^{366}$ Die Frage nach den fun-

366 Es ist nicht zuletzt unklar, welcher Parameterbereich und welches Kompaktifizierungsszenario etwa für die Reproduktion des Standardmodells Relevanz besitzen 
damentalen Entitäten des Stringansatzes findet keine eindeutige Antwort, sobald man den perturbativen Bereich der Stringtheorien überschreitet.

"The ideal definition of string/M/F/...-theory would have a sort of 'manifest duality' in which every object which could become fundamental in any limit was included as a fundamental degree of freedom. However, there is a bewildering variety of candidate fundamental degrees of freedom, especially after compactification, and such a description might well require intractable constraints among them. I One might look for some general 'principle of construction' that builds up all possible extended objects as composite objects. Perhaps the appropriate constituent degrees of freedom have not even made their appearance yet!" (Douglas (1996) 26)

Die Vielzahl der zur Disposition stehenden Kandidaten leistet konsequenterweise Spekulationen Vorschub, ob es im Rahmen des Stringansatzes (oder einer noch unrealisierten Verallgemeinerung oder Erweiterung) vielleicht fundamentalere Entitäten als Strings oder Branen gibt.

"It is likely, though, that the resolution lies in a different direction, that strings and membranes will turn out to be composites of something else. This has long been suspected in the case of string theory." (Polchinski (1996) 26)

Aufgrund der Uneindeutigkeit hinsichtlich der fundamentalen Entitäten, ist es für den Stringansatz letztlich völlig unklar, welche Strukturelemente ontisch gedeutet werden können und welche als mathematische Überschussstruktur anzusehen sind. Dies könnte einerseits als schlichte Folge der Situation zu werten sein, dass es letztlich noch nicht einmal eine ausgereifte Theorie gibt und sich die ontische Deutbarkeit in keiner Weise auf irgendeine empirische Instanz der Überprüfung dieser Theorie stützen kann. Es könnte aber, wie die zaghafte Erschliessung des nicht-perturbativen Bereichs der Stringtheorien zumindest andeutet, auch für eine vollaus-

könnten. Das heisst aber auch, dass nicht klar ist, auf der Grundlage welcher fundamentalen Entitäten die Physik des Standardmodells nach Auffassung des Stringansatzes zustandekommt:

"One might also wonder whether some or all of the Standard Model states can originate not as strings but as D-branes." (Polchinski (2000a) 343) 
gebildete Theorie bei der weiter unten zu diskutierenden Auflösung der objekthaften Ontologie bleiben.

Angesichts dieser offenen Problemlage ist es vielleicht nicht ganz abwegig, an einem Punkt zu starten, der die relevanten Fragen hinsichtlich der ontologischen Implikationen des Stringansatzes erst einmal in einer Begrenzung auf die perturbativen Stringtheorien und ihre Konzeptionen stellt, um dann, wenn es schliesslich um die Frage nach der möglichen Auflösung der objekthaften Ontologie im Stringansatz gehen wird, diesen Bereich wieder zu überschreiten.

\section{Der String als Nachfolgebegriff des traditionellen Teilchenkonzepts?}

Nehmen wir einmal an, wir hätten es mit den Strings als fundamentalen Entitäten des Stringansatzes zu tun: Kann der String unter diesen Voraussetzungen als Nachfolgebegriff des traditionellen Teilchenkonzepts verstanden werden? - Diese Frage ist nicht zuletzt schon deswegen wesentlich weniger harmlos, als sie vielleicht erst einmal klingen mag, da der Teilchenbegriff schon im Übergang von der klassischen Physik zur Quantenmechanik hinsichtlich seiner traditionellen Konnotationen erheblich relativiert wird und spätestens im Kontext der Quantenfeldtheorien kein zentraler Begriff mehr ist. ${ }^{367}$ Schon die Entwicklung der Physik vor dem Aufkommen des Stringansatzes hat den Teilchenbegriff nahezu aller klassischen Eigenschaftszuschreibungen und Substanz-Konnotationen beraubt.

"Die theoretischen Entwicklungen von der Quantenmechanik des Wasserstoffatoms bis hin zu den heutigen Quantenfeldtheorien, in denen die Beschreibung von Materiebestandteilen und Strahlungsphänomenen vereinheitlicht ist, nötigen zu immer radikaleren Abstrichen bezüglich der Prädikate, die man den Teilchen ursprünglich auf der Grundlage der klassischen Punktmechanik zuschrieb [...]." (Falkenburg (1995) 211f)

367 Die einschlägige Monographie zu den Ausprägungen und den Wandlungen des Teilchenbegriffs ist Falkenburg (1995). Siehe auch Falkenburg (1993), (1993a), (1993b), (1996), (2002b). 
Der traditionelle Teilchenbegriff umfasst im wesentlichen zwei, sich ergänzende, aber auch autonom einsetzbare Aspekte: ${ }^{368}$ Teilchen werden einerseits - im Sinne des mereologischen Teilchenbegriffs - als mikroskopische Materiebestandteile aufgefasst. Andererseits werden Teilchen - im Sinne einer kausalen Sichtweise - als mikroskopische Ursachen lokaler Wirkungen verstanden.

Beginnen wir mit einem Blick auf den kausalen Teilchenbegriff und seine Relativierungen im Rahmen der modernen Physik. Dem kausalen Teilchenbegriff liegt gleichermassen das traditionelle Kausalprinzip wie die klassische Substanzauffassung zugrunde. Die mikroskopischen Ursachen lokaler Wirkungen in einer makroskopischen Umgebung werden als substantielle Entitäten verstanden, denen als permanente Eigenschaften vor allem Masse und Ladung zugesprochen werden. Diese permanenten Eigenschaften (zu denen im Rahmen der Quantenmechanik etwa noch der Spin hinzukommt) dienen nicht zuletzt der Reidentifizierbarkeit des Teilchens. Die Dynamik des Teilchens hingegen wird auf der Grundlage seiner Dispositions- und seiner Wechselwirkungseigenschaften erfasst. Die Dispositionseigenschaften eines Teilchens schliessen insbesondere seine räumliche und zeitliche Lokalisierbarkeit sowie die Bestimmbarkeit von Impuls und Energie ein. Seine Wechselwirkungseigenschaften manifestieren sich in den Modalitäten der Ausbreitung von Wirkungen auf quasi-klassischen Raum-Zeit-Bahnen und in den jeweiligen Erhaltungssätzen, durch die sich die entsprechende Wechselwirkung kennzeichnen lässt. ${ }^{369}$

Dieser kausale Teilchenbegriff erfährt seine erste Relativierung schon in der Quantenmechanik, in deren Kontext die raumzeitliche Lokalisierbarkeit der mikroskopischen Ursachen lokaler Wirkungen in einer makroskopischen Umgebung und die vollständige Separierbarkeit ihrer als substantiell gedachten Träger in Frage gestellt werden. Eine wichtige Rolle spielen hierbei nicht zuletzt der Welle-Teilchen-Dualismus sowie die Heisenbergschen Unschärfebeziehungen.

"Bei der vollen quantentheoretischen Beschreibung von Wechselwirkungen versagt das verallgemeinerte Teilchenkonzept, das noch für die Charakterisierung der Bewegung quantenmechanischer Objekte

369 Diese Erhaltungssätze liefern eine weitere Grundlage für die Reidentifikation des Teilchens. 
unter dem Einfluss klassischer Kräfte brauchbar ist, auf der ganzen Linie. (i) Die auf der klassischen Punktmechanik beruhende Annahme einer vollständigen raumzeitlichen Determination alles dynamischen Geschehens, (ii) die dem generalisierten Teilchenkonzept zugrundeliegende vortheoretische Annahme, dass die Ursache einer lokalen Wirkung ein lokalisierbarer phänomenaler Träger permanenter Eigenschaften sein muss, und (iii) die den Gegensatz von klassischer Punktmechanik und Feldtheorie übergreifende Annahme, dass wechselwirkende Entitäten zur Beschreibung ihrer Wechselwirkung mindestens idealiter als separierbar gedacht werden können - alle diese Annahmen sind in bezug auf die Entitäten einer relativistischen Quantentheorie nicht mehr haltbar." (Falkenburg (1995) 253)

Das Kausalprinzip wird im Rahmen der durch die Schrödinger-Gleichung beschriebenen Dynamik der Quantenmechanik zwar weiterhin grundsätzlich als gültig angesehen, aber die postulierten mikroskopischen Ursachen lokaler Wirkungen in einer makroskopischen Umgebung lassen sich hier nicht mehr in irgendeiner Weise als raumzeitlich lokalisierbare und separierbare Substanzen im klassischen Sinne auffassen.

"Es gibt eine Verursachung von Teilchenspuren [...]. [Die] Ursache ist jedoch kein Teilchen im Sinne eines raumzeitlich oder dynamisch isolierten Mikroobjektes, sondern eine Entität vom Typus Prozess." (Falkenburg (1995) 260)

Wenn die Entwicklungen im Rahmen der Quantenmechanik hinsichtlich der Substantialität der mikroskopischen Ursachen lokaler Wirkungen noch Zweifel gelassen haben sollten: Im Kontext der Quantenfeldtheorien versagt der traditionelle kausale Teilchenbegriff schliesslich vollends.

"Der Teilchenbegriff kann nicht so generalisiert werden, dass er die heutigen Quantenfeldtheorien und ihre empirische Basis, die mittels der Messtheorie eines klassischen Teilchens näherungsweise erfassbar ist, zugleich abdeckt. [...] Damit zeichnet sich ab, dass man ein Teilchen nicht als etwas betrachten sollte, das lokale Wirkungen hat sondern vielmehr als etwas, das die lokale Wirkung von etwas ganz anderem als einem Teilchen ist." (Falkenburg (1995) 253) 
Die Substantialität der "Teilchen" wird nicht zuletzt durch ihre Erzeugung und Vernichtung in den Quantenfeldtheorien untergraben. Teilchen werden als Feldquanten eines fermionischen Materiefeldes aufgefasst und stehen auf der gleichen Stufe mit den bosonischen Feldquanten der Wechselwirkungsfelder.

"Wenn man ein Teilchen als eine phänomenale Substanz im Lockeschen Sinne betrachten möchte, d.h. als ein Kollektiv permanent zusammen auftretender Eigenschaften, so steht man angesichts der durch die heutigen Quantenfeldtheorien beschriebenen Phänomene vor dem Sachverhalt, dass sich Eigenschaftskollektive 'auflösen' und zu neuen Eigenschaftskollektiven 'umgruppieren' lassen [...]." (Falkenburg (1995) 252)

Aber nicht die erzeugten und wieder vernichteten Teilchen bzw. Feldquanten sind die fundamentalen Entitäten, von denen die Quantenfeldtheorien handeln; dies sind vielmehr die quantisierten Felder selbst.

"Die Teilchen, mit denen man die durch Messgeräte lokalisierbaren Feldquanten einer Quantenfeldtheorie identifiziert, stellen nicht die fundamentalen Entitäten dieser Theorie dar, sondern nur die unter bestimmten experimentellen Bedingungen realisierten nicht-permanenten Zustände einer theoretischen Entität, die als ein quantisiertes Feld mit fluktuierender Besetzungszahl konzipiert ist - was immer auch man unter einer solchen Entität genau verstehen mag. / Eine Quantenfeldtheorie ist darum keine Teilchentheorie, d.h. keine Theorie, deren Entitäten sich in irgendeinem nachvollziehbaren Sinn als lokalisierte oder lokalisierbare Teilchen auffassen lassen." (Falkenburg (1995) 249)

Vor diesem Hintergrund ist es dann auch nicht mehr so erstaunlich, dass sich im Rahmen einer quantenfeldtheoretischen Beschreibung des Vakuums für gekrümmte Raumzeiten bzw. starke Gravitationsfelder der Teilchenbegriff bzw. das Auftreten von Teilchen als beobachterabhängig herausstellt.

"Many properties of QFT which hold in Minkowski space either do not apply, or change radically on a curved background. [...] strong or rapidly varying gravitational fields can produce particles. It is easy to 
see that this makes the concept of particle ambiguous (observerdependent). [...] what the free-falling observer sees as the vacuum state of his QFT is not a vacuum state for the static observer." (Alvarez-Gaumé / Vázquez-Mozo (1992) 12)

Die Beobachterabhängigkeit manifestiert sich nicht zuletzt darin, dass ein beschleunigt bewegter Beobachter ein thermisches Teilchenbad wahrnimmt, wo ein Beobachter in einem Inertialsystem nur den Vakuumzustand des Quantenfeldes feststellen kann. Dieses Phänomen wird als "Unruh-Effekt" bezeichnet, die sich für beschleunigt bewegte Beobachter manifestierenden Teilchen als "Rindler-Quanten".

"[...] a uniformly accelerated observer will see a flux of particles as if he were in a thermal bath of scalar particles [...] while the inertial observer will see the field [...] in its vacuum state [...] and consequently will not see any particle at all. This shows that when we depart from Minkowski space the concepts of vacuum and particles become observer-dependent." (Alvarez-Gaumé / Vázquez-Mozo (1992) 15)

Spätestens diese Beobachterabhängigkeit des Auftretens von Teilchen macht deutlich, dass diesen im Rahmen der Quantenfeldtheorien nur ein ephemerer Charakter zukommt. Der zentrale Begriff bzw. die fundamentale Entität in den Quantenfeldtheorien ist eben das quantisierte Feld selbst, und nicht etwa die Teilchen.
"Wenn man [...] unter Teilchen die Referenzobjekte einer Fundamen- taltheorie der Physik verstehen möchte, die sich als so etwas wie selb- ständige 'Bausteine' der Wirklichkeit konzipieren lassen, so gibt es keine Teilchen, denn die fundamentalen Entitäten einer Quan- ten(feld)theorie der Elementarteilchen sind nie und nimmer Teilchen, sondern 'Quantenfelder', die über probabilistische Grössen mit raum- zeitlichen Phänomenen verknüpft sind." (Falkenburg (1995) 256)

Letztlich gibt es in den Quantenfeldtheorien, wenn man die Entwicklungen innerhalb ihrer algebraischen Formulierung ernst nimmt, wohl überhaupt keine lokalisierbaren Objekte im strengen Sinne mehr. ${ }^{370}$ Das Fazit kann dann nur lauten:

370 Vgl. Rovelli (1999) sowie die entsprechenden Beiträge in Kuhlmann / Lyre / Wayne (2002). 
"Nach heutigem Wissen bleibt vom kausalen Teilchenkonzept letztendlich nicht mehr viel übrig [...]." (Falkenburg (1995) 212)

Nicht viel besser ergeht es dem mereologischen Teilchenbegriff, der in noch stärkerem Masze im traditionellen Substanzbegriff verankert ist und demzufolge Teilchen als mikroskopische Materiebestandteile betrachtet werden. Konzeptionelle Grundlage des mereologischen Teilchenbegriffs ist die Teil-Ganzes-Beziehung. Dies bietet erst einmal den Vorteil, dass sich der Realstatus makroskopischer Substanzen auf ihre (im Rahmen empirisch bestätigter Theorien) postulierten mikroskopischen Konstituenten, welche auch immer dies sein mögen, zu übertragen scheint.

"Wenn ein materielles Ganzes, etwa ein Gesteinsbrocken, empirisch gegeben ist, so können seine experimentell unterscheidbaren Teile, wie gross oder klein sie auch sein mögen, nicht minder real sein: dies ist das mereologische Argument für die Existenz mikroskopischer Materiebestandteile." (Falkenburg (1995) 292f)

Existiert das makroskopische Objekt, so existieren auch seine mikroskopischen Bestandteile, soweit diese sich ausmachen lassen.

"Die Teile-Ganzes-Beziehung gestattet [...] einen bedingten Schluss von der Existenz eines Ganzen auf die Existenz seiner Teile [...]." (Falkenburg (1995) 290)

Wenn ein materielles Objekt aus mikroskopischen Konstituenten zusammengesetzt ist, so heisst dies jedoch noch nicht notwendigerweise, dass es auch in diese mikroskopischen Konstituenten zerlegbar ist und dass diese mikroskopischen Konstituenten isolierbar sein müssen.

"Die Unterstellung, Zusammengesetztsein impliziere immer auch die Teilbarkeit des Ganzen, so dass die Bestandteile eines Ganzen im Prinzip räumlich isolierbar sein müssen, hat sich jedoch als zu speziell erwiesen, um den Konstituentenmodellen der heutigen Physik standzuhalten. Auch diese Unterstellung gehört zu den bis heute nicht aus unserem Sprachgebrauch ausgerotteten Restbeständen der Substanz-Metaphysik." (Falkenburg (1995) 271) 
Die Unrichtigkeit der Annahme, dass Zusammengesetztsein notwendigerweise auch Teilbarkeit impliziert, hat sich spätestens mit dem QuarkConfinement herausgestellt. Jeder Versuch, Quarks durch Energiezufuhr aus einem Verbund zu lösen und somit zu isolieren, führt nur zu neuen gebundenen Quarkkonstellationen, nicht aber zu isolierten Quarks.

"Die Materie ist nicht beliebig separierbar in immer kleinere Bestandteile, weil im Mikroskopischen die aus dem makroskopischen Bereich vertrauten Kriterien für die Wohlunterschiedenheit oder Disjunktheit der Teile eines Ganzen zunehmend versagen." (Falkenburg (1995) 289)

Wenn aber Zusammengesetztsein nicht notwendigerweise Teilbarkeit impliziert, so zeichnet sich die Ebene der Mikrokonstituenten erst einmal nur durch eine relationale Struktur aus, welche zur Charakterisierung der Eigenschaften des zusammengesetzten Objektes beiträgt, aber nicht unbedingt dafür ausreicht, den einzelnen Mikrokonstituenten substantiellen Charakter im Sinne makroskopischer materieller Objekte zuzusprechen.

"Der Witz an der mereologischen Explikation des Teilchenbegriffs ist, dass Teilchen danach nicht als die von 'Rest der Welt' isolierten Entitäten einer fundamentalen Theorie aufgefasst werden müssen [...]. Nach der Bestehensrelation sind Teilchen soviel wie mikroskopische Bestandteile eines makroskopischen Ganzen. [...] Der Teilchenbegriff wird dadurch relational verstanden, so dass es strenggenommen sinnlos ist, von 'einzelnen' Teilchen zu sprechen. Teilchen sind [...] also keine entia per se im Sinne der traditionellen Metaphysik." (Falkenburg (1995) 284)

Im Sinne des mereologischen Teilchenbegriffs, der auf der Teil-GanzesBeziehung beruht, hängt die Zuschreibung eines ontischen Status für die postulierten Mikrokonstituenten notwendigerweise an ihrer experimentellen Erschliessbarkeit. Die experimentelle Erschliessung führt aber schon im Rahmen der Quantenmechanik nicht mehr zur vollständigen raumzeitlichen Lokalisierbarkeit der als "Teilchen" deklarierten Mikrokonstituenten der Materie. Die Komponenten eines Quantensystems sind zudem unter bestimmten Bedingungen durch EPR-Korrelationen verschränkt. Sie bilden eine korrelativ verbundene Einheit. Bestimmte quantenmechanische Eigenschaften sind nur dem gesamten System zuzuschreiben, nicht aber irgend- 
welchen Teilen des Systems. Letztlich sind es oft nicht die postulierten teilchenartigen Mikrokonstituenten selbst, die empirisch erschlossen werden können, sondern vielmehr die quantenmechanischen Systeme, in welche diese postulierten Mikrokonstituenten eingebunden sind. Ist das Kriterium das der empirischen Erschliessbarkeit, so gibt es in diesen Fällen nur die quantenmechanischen Systeme, nicht aber die postulierten Mikrokonstituenten, aus denen diese sich angeblich zusammensetzen.

Und die experimentelle Erschliessbarkeit der jeweiligen Mikrokonstituenten wird im Kontext der Quantenfeldtheorien noch problematischer. Einerseits verliert die klassische Unterscheidung in Teilchen und Felder spätestens in den Quantenfeldtheorien jegliche Bedeutung. Materieteilchen lassen sich als die Feldquanten fermionischer Materiefelder ansehen. Die Unterscheidung in fermionische Materiefelder und bosonische Wechselwirkungsfelder ist nur noch eine Frage der jeweiligen Quantenstatistik.

"Auf der untersten heute bekannten Konstituentenebene der Materie gibt es keinen willkürfreien Unterschied mehr zwischen Teilchen und Feldern, und es zeichnet sich ab, dass beim Versuch, durch Streuexperimente noch einmal kleinere diskrete Strukturen innerhalb der Materie aufzulösen, womöglich sämtliche bislang denkbaren Kriterien für Disjunktheit versagen." (Falkenburg (1995) 290)

Andererseits kommt in den Quantenfeldtheorien gegenüber der Quantenmechanik noch die Schwierigkeit hinzu, dass nicht in jeder Hinsicht klar ist, was gerade zur Konstituentenebene eines Systems zählt. Die möglichen Konstituentenkonstellationen, die nicht zuletzt vielfältige Konstellationen von virtuellen Anregungszuständen diverser Felder enthalten können, befinden sich selbst im Zustand einer quantenmechanischen Superposition.

"Ein Fermionensystem kann partiell aus Strahlungsfeldern bestehen etwa das Proton oder Neutron, das, wie die experimentelle Überprüfung der Summenregel für die Quark-Impulse zeigt, nicht nur aus drei dynamisch disjunkten Quarks besteht, sondern darüberhinaus einen Feldanteil hat, der experimentell nicht in disjunkte Komponenten separierbar ist und der erheblich zum Nukleonenimpuls beiträgt; er wird erklärt durch die Strahlungsquanten der starken Wechselwirkung, Gluonen mit Spin 1, und einen 'See' virtueller Quark-AntiquarkPaare [...]." (Falkenburg (1995) 288) 
Konsequenterweise lässt sich auch der traditionelle mereologische Teilchenbegriff hier kaum noch als angemessene Begrifflichkeit hinsichtlich einer postulierten Mikrostruktur der Materie ansehen.

"Auch der mereologische Teilchenbegriff wird [...] unscharf, wo die Kriterien für die Wohlunterschiedenheit versagen und sich der Teilchenbegriff nicht mehr gegen den Feldbegriff der Physik abgrenzen lässt." (Falkenburg (1995) 295)

Der klassische Teilchenbegriff, sowohl in seiner kausalen wie in seiner mereologischen Ausprägung, verliert also spätestens im Rahmen der Quantenfeldtheorien seine Bedeutung.

"Damit erweisen sich beide traditionellen Bedeutungen des Teilchenbegriffs, die kausale und die mereologische, letztlich als untauglich, um ein generalisiertes Teilchenkonzept zu begründen." (Falkenburg (1995) 302)

Das heisst natürlich noch nicht, dass der Teilchenbegriff im Kontext der Physik nicht mehr verwendet würde. Er wird heute gleichermassen auf die postulierten Mikrokonstituenten der Materie wie auf die Feldquanten der Wechselwirkungsfelder angewandt. Und er bleibt dabei ein schillernder, mehrdeutiger Begriff, unter dem sehr uneinheitliche, teilweise empirisch zugängliche, teilweise theoretisch postulierte Entitäten zusammengefasst werden, die in nahezu allen Fällen keine der Eigenschaften aufweisen, die Teilchen im traditionellen Verständnis zugesprochen wurden.

"Es gelang bisher nicht, ein einheitliches theoretisches Konzept der Entität 'Teilchen' zu formulieren, das hinreichend dafür wäre, alle experimentellen Befunde der Atom-, Kern- und Teilchenphysik empirisch adäquat zu beschreiben." (Falkenburg (1995) 211)

Wenn in der Physik also von "Teilchen" die Rede ist, so sind dies im allgemeinen nicht etwa raumzeitlich eindeutig lokalisierbare, mit klassischem Substanzcharakter ausgestattete, autonome oder gar isolierbare Bestandteile der Materie. - Unter diesen Vorbehalten, die sich aus der Entwicklung unseres Verständnisses der mikroskopischen Konstituentenebene der Materie (und aus der des Feldbegriffs) ergeben, kann man nun die Frage stellen, 
ob der String, zumindest im perturbativen Stringansatz, als Nachfolgebegriff zum "Teilchen" anzusehen ist?

Innerhalb des perturbativen Stringansatzes sind die "Teilchen" der Teilchenphysik die Erscheinungsformen des String. Die im Rahmen der Quantenfeldtheorien formal als punktförmig behandelten Entitäten sind dem Stringansatz zufolge eindimensional ausgedehnte Strings, die sich in einem bestimmten Oszillationszustand befinden, der sich jeweils durch bestimmte Eigenschaften (Energie bzw. Masse, Ladungen, Spin) kennzeichnen lässt. ${ }^{371}$ Mit dem Stringansatz wird also nicht etwa die bisherige, auf dem mereologischen Konstituentenbegriff beruhende Tieferlegungsstrategie atomistischer Konzeptionen weiterverfolgt, durch die sich etwa auch der Übergang von der Hadronenphysik zum Quarkmodell auszeichnete. "Teilchen" setzen sich dem perturbativen Stringansatz zufolge nicht etwa aus Strings zusammen, sondern sie sind die Erscheinungsformen des String. ${ }^{372}$ Die im Stringansatz vorgenommene strukturelle Tieferlegung ist insofern keine mereologische, sondern vielmehr eine nomologische und dynamische. Wenn man Strings gemäss den perturbativen Stringtheorien als basale Entitäten behandelt, so sind diese genau in dieser nomologisch und dynamisch verstandenen Weise als Nachfolgekonzept zum "Teilchen" zu sehen.

Der hierbei als Bezugspunkt dienende Teilchenbegriff ist aber, wie betont werden muss, der schon nicht mehr zentrale Teilchenbegriff der Quantenfeldtheorien. ${ }^{373}$ Die Strings des perturbativen Stringansatzes bringen als

371 Hierbei kommen als Oszillationszustände des String gegenüber dem Spektrum der im Rahmen der Quantenfeldtheorien erfassten Entitäten neue "Teilchen" hinzu: nicht zuletzt etwa die supersymmetrischen Partner zu den bekannten Teilchen sowie die gesamte, infinite Sequenz von Teilchen mit Massen im Spektrum von jeweils Vielfachen der Planck-Masse.

372 Das Verhältnis zwischen dem String und den "Teilchen", sowohl den bekannten als auch den neu hinzukommenden, theoretisch postulierten, lässt sich in gewisser Weise als analog zu dem zwischen einer Violinsaite und den Tönen, die mit ihr hervorgebracht werden können, charakterisieren.

"Superstring theory, crudely speaking, unites the various forces and particles in the same way that a violin string provides a unifying description of the musical tones. [...] one physical object can explain the varieties of musical notes and even the harmonies we can construct from them." (Kaku (1999) 17)

373 Die ontologischen Probleme der Quantenfeldtheorien sind jedoch noch weitgehend ungeklärt. Siehe etwa Cao (1999), Kuhlmann / Lyre / Wayne (2002) sowie Stöckler (1987). 
Zustände gleichermassen fermionische "Materieteilchen" wie bosonische "Wechselwirkungsfeldquanten" hervor. Die Strings sind damit einerseits die konzeptionellen Nachfolger der "Materieteilchen" (und indirekt somit auch des klassischen Teilchenbegriffs). Andererseits sind sie in gleicher Weise die konzeptionellen Nachfolger der Feldquanten der Quantenfeldtheorien. Schon innerhalb der Quantenfeldtheorien besteht ein Unterschied zwischen Feldern und Materie lediglich in Bezug auf die jeweilige Quantenstatistik, nicht jedoch in konzeptioneller Hinsicht. Materieteilchen und Feldquanten sind jedoch schon in den Quantenfeldtheorien nur noch - gegenüber den quantisierten Feldern - sekundäre, abgeleitete Entitäten. Sie sind gleichermassen Anregungszustände quantisierter Felder. Nun, im Stringansatz, sind sie die Anregungszustände des String. Das Verhältnis zwischen den Strings und den quantisierten Feldern als zentraler Entität der Quantenfeldtheorien bleibt jedoch im bisherigen Stringansatz weitgehend im Unklaren. Dies wird sich, wenn überhaupt, erst im Rahmen einer noch ausstehenden Weiterentwicklung klären lassen.

Die schon in den Quantenfeldtheorien bestehende symmetrische Behandlung von Materieteilchen und Wechselwirkungsfeldquanten als jeweilige Ausprägung der als basal angesehenen Materie- und Wechselwirkungsfelder, wird allerdings im Stringansatz noch einmal durch die Einbeziehung der Supersymmetrie verstärkt. Es gibt den perturbativen Stringtheorien zufolge nicht mehr die (hinsichtlich der Quantenstatistik) unterschiedlich gearteten Typen quantisierter Felder, die entweder fermionische Materieteilchen oder bosonische Feldquanten hervorbringen, sondern es gibt nur noch den String, der gleichermassen fermionische wie bosonische Zustände aufweist. Materie und Felder werden nicht nur, wie in den Quantenfeldtheorien, konzeptionell gleich behandelt, sondern sie sind dynamische Ausprägungen einer Basisentität, gehen also auf den gleichen nomologischen Ursprung zurück. Damit verliert die traditionelle Trennung von Materie und Feldern ihre Bedeutung. Die perturbativen Stringtheorien vollziehen, zumindest formal, eine gleichermassen ontologische wie nomologische Vereinigung von Materie und Feldern. ${ }^{374}$

374 Die im Stringansatz vorgenommene ontologische wie nomologische Vereinigung geht jedoch letztlich womöglich noch über Materie und Felder hinaus. Schliesslich geht es dem Stringansatz um eine nomologisch vereinheitlichte Erfassung aller Wechselwirkungen inklusive der Gravitation. Und wenn die auf die Allgemeine Relativitätstheorie zurückgehenden Einsichten hinsichtlich der Dynamizität der Raumzeit und der Identität der metrischen Eigenschaften der Raumzeit mit dem Gravitationsfeld sich schliesslich im Stringansatz niederschlagen sollten, wenn also die im Kontext der 


\section{Ontische Interpretierbarkeit des String?}

Man sollte aus der im perturbativen Stringansatz vorliegenden, nicht nur konzeptionellen, sondern ebenso dynamisch-nomologischen Zusammenführung von Materie und Feldern jedoch ganz sicher nicht darauf schliessen, dass sich der String selbst in irgendeiner Weise realistisch deuten liesse, etwa als eindimensional ausgedehnte, in ihrer Bewegung und Schwingung raumzeitlich veranschaulichbare Entität. Der String lässt sich vielmehr, wie im folgenden motiviert werden soll, bestenfalls als so etwas wie eine mathematische Metapher für die relevanten dynamischen Freiheitsgrade ansehen, aus denen gleichermassen fermionische wie bosonische Zustände einheitlich hervorgehen bzw. als einheitlich hervorgehend rekonstruiert werden können. Denn es gibt gute Gründe, die gegen eine realistische Interpretierbarkeit des String sprechen.

Würde man den String in realistischer Weise als raumzeitlich ausgedehnte, schwingende Entität verstehen wollen, so hätte dies gravierende Probleme zur Folge. Eine realistische Interpretation der Schwingungen des String müsste etwa sich bewegende Stringabschnitte ins Feld führen, also verdeutlichen, aus was der ausgedehnte, schwingende String besteht, was da also schwingt. Diese Stringabschnitte müssten zwar ganz sicher nicht isolierbar sein, aber immerhin als Konstituenten des String charakterisierbar. Es müsste, wenn der String als reale, raumzeitlich ausgedehnte Entität verstanden werden sollte, eine Beziehung zwischen dem dynamischen $\mathrm{Zu}$ sammenhalt der Stringabschnitte und den Modalitäten der Schwingung bestehen. Die Annahme der Existenz von Stringabschnitten oder Stringteilen widerspricht jedoch der Konzeption der perturbativen Stringtheorien, in-

Erörterung des Problems der Raumzeit (siehe Kap. 6.) angesprochenen Perspektiven einer Stringtheorie ohne Voraussetzung einer Hintergrundraumzeit sich schliesslich realisieren lassen sollten, so wäre der String dann vielleicht sogar als Nachfolgekonzept zur Raumzeit selbst zu sehen. Dies wäre für eine Theorie der Quantengravitation nur konsequent. Denn, wenn man das Gravitationsfeld mit den metrischen Eigenschaften der Raumzeit identifiziert, wenn sich diese also auf Gravitationsfelder zurückführen lassen, und wenn Gravitationsfelder durch Gravitonen realisiert werden, und wenn Gravitonen Oszillationszustände von Strings sind, so müssten sich die metrischen Eigenschaften der Raumzeit - wenn nicht die Raumzeit selbst - konsequenterweise auf Strings zurückführen lassen. 
nerhalb derer der String als basale, irreduzible Entität behandelt wird. Als basale, irreduzible Entität kann der String per se nicht als im raumzeitlichen, realistischen Sinne ausgedehnt verstanden werden, sondern nur als ein dynamisches Etwas mit bestimmten Eigenschaften, die sich in gewisser Weise so verhalten wie eine quantisierte Variante einer klassischen ausgedehnten Saite.

Zudem: Wie sollten realistisch, im raumzeitlichen Sinne, verstandene Stringoszillationen auch empirisch erschlossen werden und damit ihre realistische Deutbarkeit erhärtet werden? Wie sollte eine räumliche Stringauslenkung empirisch festgestellt werden? Etwas, was die räumlichen Teile eines String in ihrem dynamischen Verhalten untersuchbar werden liesse, müsste kleiner und energiereicher als der String selbst sein. Zumindest im Rahmen der perturbativen Stringtheorien gehören Strings aber per se dem Bereich kleinster räumlicher Abstände an. Es gibt für die perturbativen Stringtheorien eine kleinste räumliche Distanz, die sich an der Stringlänge orientiert. ${ }^{375}$ Für diese kleinste räumliche Distanz spricht nicht zuletzt auch die T-Dualität. ${ }^{376}$ Eine empirische Erschliessungsmöglichkeit, auf die sich eine realistische Interpretation der Stringdynamik stützten könnte, müsste diese kleinste Länge unterlaufen. Damit ist eine solche Untersuchungsmethode und somit eine realistische Interpretation des String als schwingendes Objekt mit der Konzeption der Stringtheorien inkompatibel.

Letztlich benötigt man diese Inkompatibilität einer realistischen, raumzeitlichen, objekthaften Interpretatation des Strings mit der Konzeption der perturbativen Stringtheorien jedoch gar nicht, um für den Verzicht auf erstere zu plädieren. Denn es gibt ein weiteres durchschlagendes Argument, das - völlig unabhängig vom Stringansatz und seinen spezifischen Konstruktionen - eine Erschliessung von Längenintervallen unterhalb der Planck-Länge unmöglich erscheinen lässt:

"If we want to explore space with resolution of order $r$, the uncertainty principle tells us that we need to use energy $E>1 / r$. This energy has to be concentrated in a region of the size r. But in the presence of gravitational interactions, this concentration of energy creates a black

375 Gedankenexperimente, die auf die Unmöglichkeit der Erschliessung von Grössenordnungen unterhalb der Stringlänge hinweisen, finden sich etwa in Amati / Ciafaloni / Veneziano (1989) sowie in Calmet / Graesser / Hsu (2005).

376 Siehe Kap. 2.3. 
hole unless $r>l_{P}$. Therefore, we cannot explore distances smaller than the Planck length." (Seiberg (2006) 5f)

Alles, was sich in der Grössenordnung der Planck-Länge abspielt (die im Sinne der perturbativen Stringtheorien mit der Stringlänge in etwa identisch ist), mag zwar basal sein, ist aber nicht mehr realistisch deutbar im Sinne eines Verhaltens und Interagierens von raumzeitlich ausgedehnten und raumzeitlich charakterisierbaren Entitäten. Es kann für diesen Bereich keinen Objektbegriff mit raumzeitlichen Konnotationen mehr geben.

Der Stringansatz handelt also bestenfalls von einer mathematischen Struktur, auf deren Grundlage sich zwar vielleicht raumzeitliche Objekthaftigkeit und vielleicht sogar Raumzeit konstituieren könnten, der aber selbst diese Eigenschaften ganz sicher nicht zukommen. Strings können keinesfalls als räumlich strukturierte und ausgedehnte Entitäten verstanden werden. Sie stehen vielmehr - zumindest, wenn man die perturbativen Stringtheorien konzeptionell ernst nimmt - als strukturelle Metapher für ein dynamisches Etwas, das auf der Grundlage seiner Freiheitsgrade durch seine verschiedenen dynamischen Zustände auf spezifische Art und Weise kausal wirksam wird, ohne dass ihm selbst in irgendeiner Weise schon Objekthaftigkeit zugesprochen werden könnte.

\section{Auflösung der objekthaften Ontologie}

Nachdem das Konzept objekthafter Mikrokonstituenten des materiellen Geschehens schon mit der Quantenmechanik und erst recht mit den Quantenfeldtheorien nahezu alle traditionellen, klassischen Konnotationen verloren hat, erfolgt im Stringansatz der endgültige Schritt zu einer Auflösung jeglicher objekthaften Ontologie.

"I think that the full dissolution of ontology is a characteristic process of particle physics whose unfolding starts with quantum mechanics and gains momentum in gauge field theory until, in string theory, the ontological object has simply vanished." (Dawid (2003) 23) 
Insbesondere mit dem Übergang von den perturbativen Stringtheorien zu ihren nicht-perturbativen Erweiterungen wird die schon für den perturbativen String zu konstatierende (und vorausgehend beschriebene) Auflösung der objekthaften Ontologie noch ausgeprägter. Hier gibt es nun auf einmal völlig unterschiedliche Beschreibungsweisen, die z.T. gänzlich verschiedene (basale?) Entitäten postulieren, die aber hinsichtlich ihrer physikalisch relevanten Implikationen äquivalent sind. Dies wird vor allem in den diversen Dualitäten deutlich - den Identitäten der Zustandsspektren für völlig unterschiedliche theoretische Szenarien mit gänzlich unterschiedlicher, vermeintlich ontologischer Festlegung. ${ }^{377}$ So führt die T-Dualität zwischen den Theorien vom Typ IIA und vom Typ IIB zu einer Äquivalenz zwischen einer chiralen und einer nicht-chiralen Theorie. Die Spiegelsymmetrie impliziert eine Äquivalenz von theoretischen Szenarien, die sich auf der Grundlage unterschiedlicher Raumzeittopologien ergeben. Im Rahmen der S-Dualität kommt es schliesslich für bestimmte Parameterbereiche zur Äquivalenz zwischen theoretischen Szenarien mit offenen und solchen mit geschlossenen Strings. ${ }^{378}$ Ebenso stellt die S-Dualität Äquivalenzen zwischen theoretischen Konstrukten her, die mit unterschiedlicher Dimensionalität der Raumzeit arbeiten. ${ }^{379}$ Letzten Endes bleiben nicht einmal die Begrifflichkeiten der "starken" und der "schwachen" Kopplung eindeutig:

"The lesson is that one and the same physical theory can have many perturbative descriptions. These may look completely different, and can involve different gauge groups and matter fields. There is in general no absolute notion of what would be weak or strong coupling; rather what we call weak coupling or strong coupling, or an elementary or a solitonic field, depends to some extent on the specific description that we use. Which description is most suitable, and which physical degrees of freedom will be light or weakly coupled (if any at Siehe Kap. 2.3.

So entspricht der Bereich der starken Kopplung der Typ-I-Theorie, welche die Dynamik von unorientierten offenen Strings erfasst, der schwachen Kopplung für die heterotische SO(32)-Theorie, die ausschliesslich das Verhalten von orientierten geschlossenen Strings beschreibt.

379 So entspricht der Bereich der starken Kopplung sowohl der zehndimensionalen Typ-IIA-Stringtheorie als auch der (ebenso zehndimensionalen) heterotischen $\mathrm{E}_{8} \mathrm{x}$ $\mathrm{E}_{8}$-Stringtheorie gleichermassen dem Bereich der schwachen Kopplung der elfdimensionalen Supergravity.
} 
all), depends on the region of the parameter space we are looking at." (Lerche (2000) 23)

Die sogenannte "Ladungs-Ausdehnungs-Dualität" impliziert schliesslich die Abhängigkeit der Wechselwirkungsgrössen ("Ladungen") von der Grösse der kompakten Dimensionen. Die auch als "Dualitätsdualität" bezeichnete U-Dualität führt auf einer höheren strukturellen Abstraktionsebene zur wechselseitigen Übersetzbarkeit zwischen der T-Dualität für solitonische Strings und der S-Dualität für die gewöhnlichen fundamentalen Strings der perturbativen Stringtheorien. ${ }^{380}$ Die Dualitäten zwischen unterschiedlichen theoretischen Szenarien lassen sich, wie sich hier zeigt, als Austausch der als fundamental behandelten Entitäten zwischen jeweils zwei Beschreibungsweisen interpretieren.

"There is some confusion, however, over which extended objects are 'fundamental' and which ones are 'solitonic', which differ by whether they are divergent at the origin. Under a duality transformation, in fact, we can turn one into the other." (Kaku (1999) 517).

Spätestens diese weitgehende Flexibilität (wenn nicht gar Beliebigkeit) in der Wahl der Basisentitäten macht die Zuschreibung eines ontisch reliablen Status für die jeweils gerade postulierten Basisentitäten völlig unglaubwürdig.

"[...] what is fundamental and what is solitonic is a matter of taste." (Kaku (1999) 517).

Die in den diversen Szenarien postulierten Entitäten (Strings, Branen diverser Dimensionalität) können - genausowenig wie die ihnen zugesprochenen dynamischen Eigenschaften und die jeweils zugrundegelegten raumzeitlichen Kontexte (Kompaktifizierungsmodi) - realistisch gedeutet werden. Sie können nicht als objektartig verstanden werden, sondern lediglich als unter verschiedenen parametrischen Bedingungen austauschbare strukturelle Metaphern, die - den empirisch untermauerbaren Erfolg des theoretischen Ansatzes einmal angenommen - das Auftreten einer Typologie physikalisch relevanter, kausaler Wirkungsmöglichkeiten erfassbar werden lassen könnten. Die Festlegung auf bestimmte Basisentitäten lässt sich jedoch bestimmt nicht als ontologische Festlegung des Stringansatzes

$380 \quad$ Siehe Schwarz (1997) und Duff (1998). 
deuten; die basalen Entitäten der jeweiligen theoretischen Szenarien haben lediglich den Status einer für das jeweilige Szenario (und den von ihm erfassten Parameterbereich) relevanten, basalen mathematischen Struktur.

"String theory simply is no theory about invisible external objects." (Dawid (2003) 28)

Nicht einmal die Festlegung dessen, was über die spezifischen theoretischen Szenarien hinweg als Materie, als Wechselwirkung und als Komponente der Raumzeit angesehen wird, bleibt im Kontext der Dualitätsbeziehungen eindeutig:

"[...] which fields are 'matter' and which ones are 'geometrical' is subjective, depending on whether you adopt an M theory or IIB theory viewpoint. Both viewpoints are valid, and neither is preferable to the other. So how many compact dimensions there are is just a matter of how the fields are labelled!" (Schwarz (1997) 27)

Felder, die innerhalb eines theoretischen Konstrukts als metrische Komponenten in Erscheinung treten, entsprechen Materiefeldern in dem $\mathrm{zu}$ ihm dualen Konstrukt. Welche Felder als interne Komponenten des metrischen Tensors zu sehen sind und welche als Materiefelder, ist also offensichtlich reine Interpretationssache. - Aber die raumzeitlichen Szenarien sind im Stringansatz ohnehin auch schon unabhängig von dieser Problematik in ihrer Festlegung uneindeutig, da die Beziehung zwischen der zehndimensionalen Beschreibung der perturbativen Stringtheorien und der ViererRaumzeit der Phänomenologie sich grundsätzlich gleichermassen im Rahmen des Kompaktifizierungskonzepts wie in dem des Bran-Ansatzes herstellen lässt.

"[...] there are currently two main approaches (related by duality) for obtaining standard and non-standard field theories from string theory [...]. One can either study the singular geometry of Calabi-Yau or other compactification manifolds, and consider the effect of wrapped branes [...]. / Alternatively, on can model the relevant string geometry in terms of parallel D-branes, with open strings and other branes running between them [...]." (Lerche (1997) 14) 
Auch wenn man sich auf das Kompaktifizierungskonzept beschränkt, ist immer noch unklar, ob die als kompaktifiziert angesehenen Dimensionen tatsächlich als raumzeitlich oder als intrinsische Freiheitsgrade der Dynamik zu deuten sind.

"It is most likely irrelevant whether these extra dimensions exist in any literal sense. If one is drawn to a picture of our three-dimensional 'reality' embedded in some higher-dimensional realm, then one can believe in the extra dimensions, at least as long as one is working in this background dependent picture. But these extra dimensions can also be seen as purely theoretical devices which are useful for understanding the list of consistent string theories in three dimensions." (Smolin (2000) 160)

Schon für die heterotischen Stringtheorien werden die über die zehn Dimensionen des perturbativen Superstringansatzes hinausgehenden 16 bosonischen Dimensionen gemeinhin nicht mehr raumzeitlich gedeutet, sondern als intrinsische Freiheitsgrade. Sie werden also nicht als physikalisch real behandelt, sondern als modelltheoretisches Instrumentarium zur Implementierung der dynamischen Freiheitsgrade des heterotischen String. Und für die sechs bzw. sieben zusätzlichen Dimensionen der perturbativen Superstringtheorien bzw. der angezielten nicht-perturbativen M-Theorie könnte das gleiche gelten. Oder es wäre denkbar - wie einige Stringtheoretiker spekulieren -, dass es sich bei diesen zusätzlichen Dimensionen weder um räumliche noch um zeitliche handelt, sondern um irgendeine bisher noch nicht in Erscheinung getretene dritte Kategorie von "geometrischer" Dimension. Die Stringtheorien selbst legen keine eindeutige Interpretation hinsichtlich dieser Problematik nahe.

Und spätestens angesichts des virulenten Problems der Hintergrundraumzeit $^{381}$ ist die realistische Deutung des Bildes der Raumzeit, wie es sich innerhalb des Stringansatzes darstellt, ohnehin äusserst fragwürdig.

"In its current stage of development, string theory unfortunately provides little indication of the more fundamental nature of space, time, and matter. Despite the consideration of ever more exotic objects strings, p-branes, D-branes, etc. - these objects are still understood as propagating in a background spacetime. Since string theory is suppo-

$381 \quad$ Siehe Kap. 6. 
sed to describe the emergence of classical spacetime from some underlying quantum structure, these objects are not to be regarded as truly fundamental. [...] Thus although string theory purports to be a fundamental theory, the ontological implications of the theory are still obscure." (Weinstein (2005) 8)

Ob jedoch die für den Stringansatz zu konstatierende Auflösung einer objekthaften und raumzeitlich deutbaren Ontologie als notwendige Konsequenz einer der Naturbeschreibung angemessenen Entwicklung der Physik anzusehen ist, oder vielmehr als Artefakt einer fehlgeleiteten Theorienbildung, bleibt vor dem Hintergrund des ungeklärten Status des Stringansatzes reine Spekulation. Die Verhandlung hinsichtlich der ontologischen Implikationen des Stringansatzes kann schon deswegen zu keinem endgültigen Ergebnis führen, als nicht klar ist, ob dieser schliesslich an seinen Problemen (fehlendes physikalisch motivierbares Prinzip, Problem der Hintergrundraumzeit, Kontingenzproblem etc.) scheitern wird, oder, ob er weitere Metamorphosen durchlaufen wird und entsprechende konzeptionelle Verschiebungen erfahren wird, in deren Kontext seine momentanen Problemlagen sich als lösbar erweisen. 